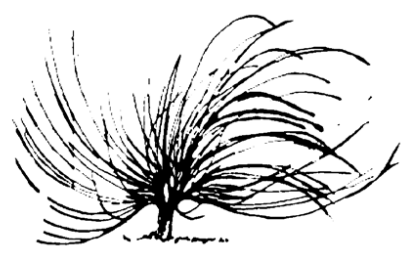

\title{
La práctica de escritura en la universidad hondureña
}

\author{
Flora Raquel Medina ${ }^{l}$ \\ Universidad Pedagógica Nacional Francisco Morazán \\ Honduras \\ floramedina24@hotmail.com \\ Lily Cálix ${ }^{2}$ \\ Universidad Pedagógica Nacional Francisco Morazán \\ Honduras \\ lilycalix@yahoo.com
}

\begin{abstract}
Selma Regina Olla Paes de Almeida ${ }^{3}$
Universidade de São Paulo

Brasil

selma.almeida@usp.br
\end{abstract}

\begin{abstract}
Resumen
En este artículo se abordarán las prácticas de escritura en la universidad hondureña por medio del análisis de algunos trabajos presentados por estudiantes de práctica profesional a través de los diarios de campo. Los resultados se sustentan en el análisis de treinta escritos de dicentes de tres carreras de formación docente y la revisión se enmarcó a nivel institucional. Este estudio forma parte de la investigación interinstitucional financiada por CAPES-Brasil, "La escritura sobre las prácticas de enseñanza en licenciaturas de Brasil, Costa
\end{abstract}

http://dx.doi.org/10.15359/rep.esp-20-1.13

1 Flora Raquel Medina Zavala es profesora universitaria y doctoranda por la Universidad Pedagógica Nacional Francisco Morazán.

2 Lily Yanet Calix Rodríguez es profesora universitaria y máster por la Universidad Pedagógica Nacional Francisco Morazán.

3 Selma Regina Olla Paes de Almeida es profesora y mestranda por la Universidade de São Paulo. 
Rica y Honduras: Registro, análisis y producción de conocimiento". A lo largo de este artículo buscaremos exponer la problemática que, de manera particular, presentan las universidades involucradas, además de recoger inquietudes e interrogantes, en relación con los procesos de lectura y escritura en educación superior.

Palabras clave: Escritura, práctica profesional, pregrado, educación superior.

\begin{abstract}
This article will address the writing practices in a Honduran university through the analysis of some papers turned in by students of professional practicum as field diaries. The results are based on the analysis of thirty written statements from three courses of teacher training, and the review was framed at institutional level. This study is part of an inter-institutional research funded by CAPES-Brazil, "Writing about teaching practices in bachelor degrees in Brazil, Costa Rica, and Honduras: register, analysis, and production of knowledge." Throughout this article, we will seek to describe the particular problem that the universities involved have in addition to seeking to raise concerns and questions regarding the processes of reading and writing in higher education.
\end{abstract}

Keywords: writing, practicum, undergraduate courses, higher education

\title{
Introducción
}

$\mathrm{L}$

a escritura se ha constituido en uno de los medios de comunicación más antiguos, por ello se convierte en una práctica habitual para la humanidad. Representa para el personal docente un gran reto, debido a las exigencias que se presentan en el siglo XXI, por lo tanto corresponde a la universidad formar profesionales de la docencia que respondan a las exigencias de esta sociedad del conocimiento que, según López y Arciniega (2007, p. 1): “exige el manejo apropiado de una diversidad de textos con altos grados de abstracción y de complejidad 
creciente, como también una gran capacidad crítica y analítica para transformar y construir conocimiento".

En cuanto al estudiantado universitario, pareciera ser que aún asumen la lectura y la escritura como simple tarea escolar: leen y escriben por razones prácticas o instrumentales, para satisfacer los requerimientos del profesorado o para alcanzar una nota, obtener un diploma etc.; situación, muchas veces aceptada por una parte del profesorado, a quienes parece no preocuparles y que, incluso, la propician.

De acuerdo con Carlino (2004), el estudiantado enfrenta cuatro dificultades en el proceso de escritura académica. El primero es que no piensan, al escribir, quiénes leerán sus textos, por lo general su escrita es basada en el autor (Flower, 1979, en Carlino, 2004) es decir, el estudiantado evidencia las informaciones comprendidas de acuerdo con el orden en que fueron identificadas, reproducen el proceso de pensamiento de los escritores o escritoras, no hay una reordenación del texto con el propósito de comunicarse con el público lector.

La segunda dificultad que se desarrolla a causa de no pensar en el sujeto lector es que no se aprovecha la potencialidad de escribir y, cuando se hace, se restringe a explicar qué se comprendió sobre el tema en cuestión, sin analizar qué se pretende con su escritura, ejercicio que permite resignificar el conocimiento anterior.

La tercera debilidad que apunta la autora es que la revisión que hace el estudiantado del texto es superficial, revisa solamente los aspectos "estilísticos" como palabras o frases, pero no el texto como un todo llevando en cuenta la modificación de las perspectivas y de las ideas.

La última dificultad que tiene el estudiantado en el proceso de escritura académica es que es el momento de comenzar el acto de escribir es postergado, es decir, se apropian de mucha bibliografía, leen bastante, pero no escriben los pensamientos e ideas que les vienen, tampoco lo que pretenden producir en su texto. Se restringen a escribir una interpretación de lo que afirman las fuentes consultadas y no repiensan lo escrito buscando independizarse de lo leído.

Por lo antes expuesto, la Universidad Pedagógica Nacional Francisco Morazán, como responsable de formar docentes, está interesada en realizar investigaciones tanto a nivel institucional como interinstitucional en proyectos que conlleven a la alfabetización académica, que corresponde a los modos de leer y escribir propios de las distintas áreas del conocimiento. 
Por ello, realizamos el presente estudio cuyo objetivo es analizar e interpretar las prácticas de escritura en universidad hondureña con el propósito de conocer cómo se producen conocimientos.

\section{Lectura y escritura en el contexto universitario}

El estudiantado que ingresa al sistema educativo formal desde el nivel prebásico es preparado para entrar al proceso de lectura-escritura, cada nivel de escolaridad que le recibe evalúa su alfabetización; sin embargo, si el resultado final de este proceso presentara algunas debilidades, sus responsables se vuelven anónimos ante ese juicio.

Es importante reconocer que en educación básica y media es la Secretaría de Educación Nacional quien da las normas de cómo debe orientarse este proceso pedagógico al interior del aula, da las pautas al personal docente acerca de qué y cómo enseñar a leer y a escribir. En este sentido, las universidades tienen mayor libertad para efectuar mejoras y, por lo tanto, rediseñar su currículo.

Por todo esto, creemos que corresponde a la educación superior contrarrestar aquellas deficiencias que presenta el estudiantado de primer ingreso a la universidad, en relación con la lectura y escritura. De acuerdo con Cisneros, Olave y Rojas, "la educación superior, entonces, tiene la gran responsabilidad en la formación lectora a un nivel avanzado, que le permita al estudiante no solo asimilar información con base en el texto escrito, sino también abrir la puerta hacia procesos de investigación y resolución de problemas" $(2013$, p. 6).

Es decir, lo que señalan como responsabilidad de la formación lectora más avanzada es, justamente, uno de los objetivos de la universidad frente a sus estudiantes: conducirles a que, por intermedio del conocimiento, avancen en investigaciones y resoluciones de los problemas existentes en nuestra sociedad.

Para ello, la escritura y la lectura son un eje transversal en la formación del estudiantado universitario. Por cuanto, leer y escribir en la universidad debe ser una actividad de enseñanza - aprendizaje infaltable en la planificación de las asignaturas, sin importar si esta es de formación general, pedagógica o de especialidad, por los consiguientes beneficios que tendrán en la formación de los futuros grupos profesionales en su desempeño.

En este contexto, emerge la necesidad de que el alumnado desarrolle un trabajo significativo, práctico, cooperativo e independiente 
para estimular el deseo por aprender, para que pueda organizar sus ideas y comunicar sus pensamientos. La importancia de la lectura y la escritura es tal que la productividad científica y la pertenencia a la vida académica se relacionan directamente con las capacidades de lectura y escritura (Pérez y Rodríguez, 2013), por lo que el desarrollo de la competencia textual discursiva y la competencia comunicativa es fundamental en la formación de un sujeto universitario para un efectivo desempeño en el campo laboral.

$\mathrm{Al}$ ingresar a la universidad, el estudiantado enfrenta un tipo de escritura diferente de la que estaba familiarizado, los textos con los cuales pasa a relacionarse tienen carácter científico y académico, dialogan con un público lector conocedor del tema y del campo de estudio a que se refieren, es decir, son textos dirigidos a personas académicas, además de contener saberes que el alumnado aún no maneja, lo que le exige un cambio para la adecuación a esa nueva manera de relacionarse con los textos y con el conocimiento que es compartido en la academia. Ese cambio presupone una especificidad en lidiar con esos textos, tal competencia exige la universidad a sus estudiantes, pero no les enseña cómo adquirirla. La nueva cultura escrita no le ha sido presentada anteriormente y al conocerla, necesita relacionarse con ella de una forma que hasta entonces no le era sabida, y resulta que muchas veces no tiene una orientación de cómo y qué hacer para lograr una mejor inserción (Carlino, 2003a).

En la práctica, este enfoque exige profesorado universitario convencido de que la escritura no es algo opcional o marginal a su ejercicio docente, sino una competencia necesaria para el desarrollo y la comunicación del pensamiento. Un trabajo así representa cooperación absoluta entre el personal docente de una institución universitaria, el cual deberá concebir la lectura y la escritura como vínculos estrechos en sus actividades académicas y, por ende, mecanismo que se adhiere a sus disciplinas, y a las necesidades e intereses de la población universitaria.

\section{Diario de campo}

La práctica educativa reflexiva constituye una estrategia fundamental para el desarrollo y para la formación profesional de estudiantes de pregrado y para la formación del profesorado en general, constituida por diversos tipos de acciones que tienen la intencionalidad explícitamente educativa y produce transformación de quienes colaboran. 
Las universidades consideran, dentro del programa de la formación docente, la realización de una práctica profesional supervisada; esta experiencia tiene en común el trabajo con docentes en servicio, a lo que normalmente se le llama profesorado titular. Una de las principales tareas que realiza el profesorado para recuperar su práctica son los autorregistros, en este caso el diario de campo, en el cual anotan lo que observan y vivencian durante el período de práctica en la escuela y las dificultades que enfrentan a lo largo de esta experiencia.

Los diarios constituyen un instrumento técnico utilizado en investigación, además se convierten en un "recurso de reflexión sobre la propia práctica profesional y, por tanto, en instrumentos de desarrollo y mejora de uno mismo y de la práctica profesional que ejerce" (Zabalza, 2004, p. 11). Este recurso es utilizado por el estudiantado durante el período de la práctica profesional. Le sirve como aparato para hacerse más consciente de su experiencia en el centro educativo, sea este de prebásica, básica o nivel medio, ya que por cuatro meses durante el año acude a realizar sus prácticas profesionales, lo cual le permite entrar en contacto con niños, niñas y adolescentes. Lo que aprendieron de los libros y las explicaciones de las clases en estas prácticas se hace realidad.

En los diarios de campo que fueron analizados, el estudiantado registraba lo que había hecho el día de la práctica y cómo se sintió, pues estas eran las dos preguntas disparadoras. De acuerdo con Moraes y Fairchild (2018), la escritura en la práctica debe conducir a la reflexión del profesorado en formación respecto de su práctica más allá de lo habitual. Hay que tener en cuenta la escritura como un proceso y por ello apuntar lo que se debe especificar.

Cisneros, Olave y Rojas (2009, p. 17) opinan que "el egresado requiere de capacidad investigativa que promueva el diálogo y la construcción de saberes para seguir aportando a la construcción de su comunidad académica". Muchas veces la práctica educativa que realiza el estudiantado está impregnada de normatividad técnica y de la exigencia (de cada docente en función asesora o bien del personal administrativo educativo) para cumplir con las normas. Sin embargo, ninguna normatividad conduce por si misma a verificar tangiblemente la intelección humana, si quien intelige, el sujeto en formación, no da cuenta de ella. 


\section{Metodología y análisis}

En relación con la metodología utilizada, la investigación se basó en un análisis cualitativo de carácter descriptivo interpretativo; se consideraron los diarios de campo de estudiantes que cursaron el espacio de aprendizaje llamado Práctica Profesional II.

Para la transcripción de los textos, este estudio se basó en los principios de la crítica textual (Spina, 1994) y en su rigor filológico para tratamiento del corpus. Se presentan los textos originales en edición fac-similar seguidos de la transcripción diplomática de ellos. A fin de evitar intervenciones del sujeto investigador en el texto original y con miras a realizar una transcripción fiable de los manuscritos para posterior análisis, se buscó el tratamiento del corpus que compone este artículo, la máxima fidelidad a fin de mantener el texto en su forma original y lo más próximo al escrito por sus autores y autoras.

La valoración de los escritos estudiantiles a nivel institucional fue realizada por personal docente involucrado en el proyecto de investigación y además en representación de las carreras estudiadas, este análisis fue desarrollado de manera conjunta. Para la valoración de los escritos se consideraron dos categorías: el aspecto conceptual y el estratégico. El análisis de las producciones escritas permitió identificar las dificultades y los logros más significativos del estudiantado, es decir, aquellos aspectos que se presentaban con mayor frecuencia en los trabajos escritos e informes finales.

Al observar los diarios, notamos que muchos, de hecho, siguen las preguntas disparadoras y contestan qué han hecho el día de la práctica y cómo se sintieron. Para una experiencia inicial, esas preguntas pueden auxiliar a que el estudiantado ingrese en su proceso de escritura, pero es necesario que el personal docente avance en las orientaciones a fin de conducirlo escrituras más específicas que dialoguen con el saber científico que proporciona la universidad para la construcción de saberes que su práctica le proporciona.

A continuación se presentan las reproducciones de algunos de los textos de estudiantes por medio de la reproducción fac-similar, seguidas de las transcripciones y de los análisis: 
Flora Raquel Medina - Lily Cálix - Selma Regina Olla Paes de Almeida

Figura 1. Reproducción fac-similar de parte del diario de campo producido por una estudiante de la Práctica

Lunes 9 de Jinio 2014. ¿Que hice?

Ture clase con Electricidad, les lleve in video, qe era introduccion de la clase, sin embargo analizor es video tomo 15 mins. más de 10 previsto. pues hizo que muchos comenzaren a hablar del tema. El vides se Hamabe Personas qe han salido adelonte pese a la adversidad".

Luego solicité a los alumnos cualquir trabajo pendiente pues dibia pasar notas.

Transcripción del texto 1, producido por estudiante de Práctica II (en la Universidad Pedagógica Nacional Francisco Morazán, Tegucigalpa, Honduras)

||Txt.1. || Lunes 9 de Junio 2014. | ¿Que hice? | Tuve clase con Electricidad, les lleve | un video, que era introducción de | la clase, sin embargo analizar el $\mid$ video tomo 15 mins. más de lo | previsto. pues hizo que muchos comenzaron | a hablar del tema. El video se llamaba | "Personas que han salido adelante pese a | la adversidad." | Luego solicité a los alumnos cualquier | Trabajo pendiente pues debía pasar | notas.

La primera frase del texto no deja claro qué significa tener clases con electricidad: ¿Será que la escuela donde la estudiante hace la práctica suele no tener energía eléctrica y al día del relato sí hubo, o si no,

$276 \cdot$

Revista Ensayos Pedagógicos

Edición Especial, 2020

269-288, ISSN: 1659-0104, EISSN: 2215-3330 
cómo ella iba a presentar un video, o en su aula hubo electricidad, pero en otras no?

La estudiante afirma que presentó un video e informa su título, pero no hay la descripción de lo que trata el video, tampoco el tema de la clase y la relación de este con lo que fue dado en sala. Aunque la estudiante haya escrito que la actividad tomó 15 minutos más de lo previsto a causa de la mucha participación de sus estudiantes, ella no especifica cómo fue esa participación, sobre qué hablaron o algo que indique más detalles de la actividad.

Figura 2. Reproducción fac-similar de parte de diario de campo producido por una estudiante de la Práctica

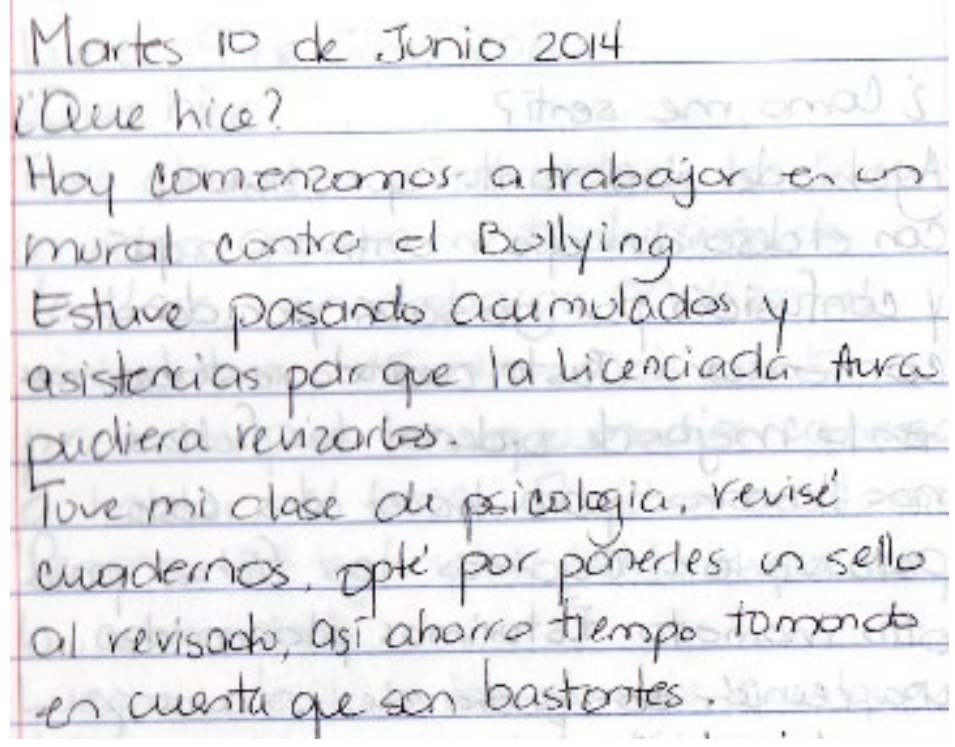

Transcripción del texto 2, producido por una estudiante de la Práctica II (en la Universidad Pedagógica Nacional Francisco Morazán, Tegucigalpa, Honduras)

||Txt.2. || Martes 10 de Junio 2014. | ¿Que hice? | Hoy comenzamos a trabajar en un| mural contra el Bullying | Estuve pasando acumulados y $\mid$ asistencias por que la Licenciada Aura $\mid$ pudiera revisarlos. | Tuve mi clase de psicología. revisé | cuadernos. Opté por 
ponerles un sello $\mid$ al revisado, así ahorro tiempo tomando | en cuenta que son bastantes.

En este pasaje se nota solamente un listado de actividades que ha hecho ella ese día. No hay una descripción más minuciosa de ninguna de estas.

Los próximos dos textos serán analizados conjuntamente porque el texto 4 es continuación del tercero:

Figura 3. Reproducción fac-similar de parte de diario de campo producido por una estudiante de la Práctica

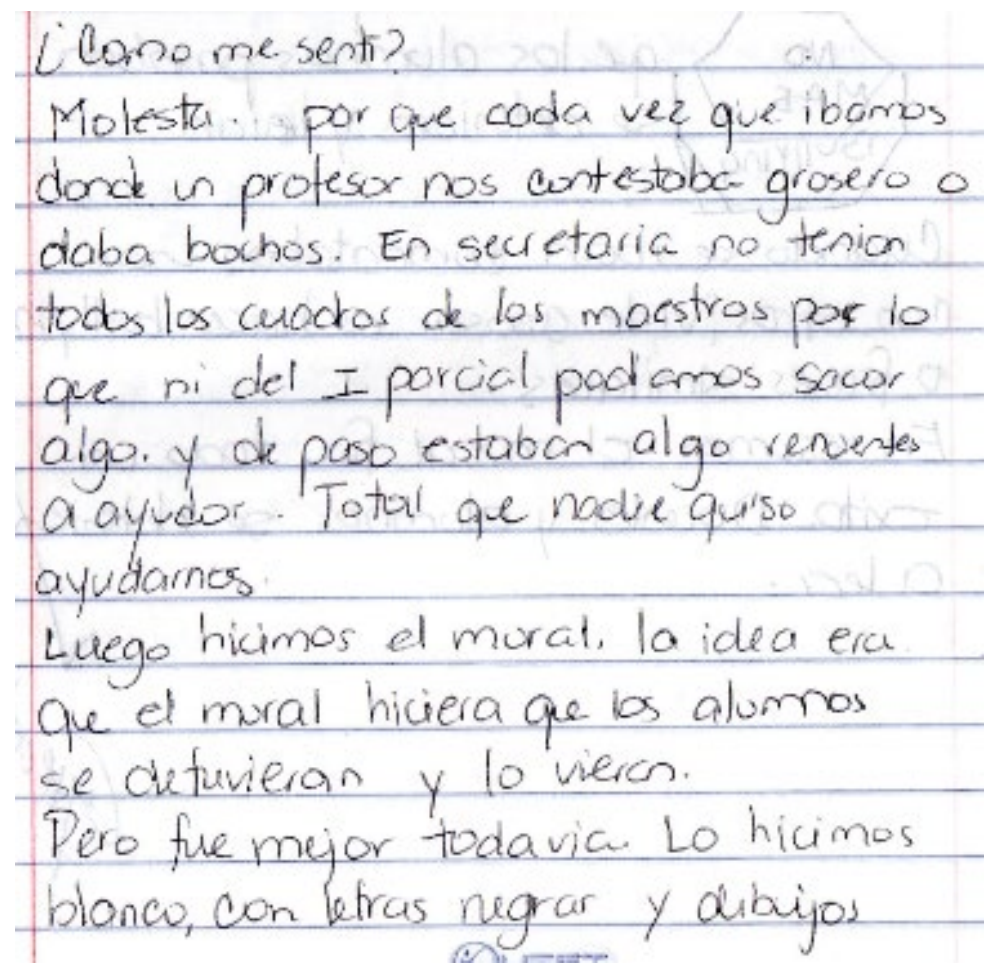

Transcripción del texto 3, producido por estudiante de la Práctica II (en la Universidad Pedagógica Nacional Francisco Morazán, Tegucigalpa, Honduras) 
||Txt.3. || ¿Cómo me sentí?| Molesta ... por que cada vez que ibamos $\mid$ donde un profesor nos contestaba grosero o $\mid$ daba bochos. En secretaria no tenian | todos los cuadros de los maestros por lo | que ni del I parcial $\quad$ podiamos sacar $\mid$ algo. Y de paso estaban algo renuentes $\mid$ a ayudar. Total que nadie quiso | ayudarnos $\mid$ Luego hicimos el mural. La idea era $\mid$ que el mural hiciera que los alumnos $\mid$ se detuvieran y lo vieran. | Pero fue mejor todavia Lo hicimos | blanco, con letras negras y dibujos

Figura 4. Reproducción fac-similar de parte de diario de campo producido por una estudiante de la Práctica

sin color, solo la silueta negra. conen tagono tipo de "alto" color rojo. que decia en letras bloncas "No mas Bullying"

Ast como cuando vemos una señal de atto, ain sn saber leer, quien ve esa señal se detiene. Asi qu cada ver No qe los alumnos pasabon MAS BullYing se detenian y leian.

Cuando se iban comentabon wos con otros "viste acosar es hacer bullying" 0 frases similares En resumen el mural fie todo in exito. Docentes y alumnos se detrich a leer. 


\section{Transcripción del texto 4, producido por una estudiante de la Prác-} tica II (en la Universidad Pedagógica Nacional Francisco Morazán, Tegucigalpa, Honduras)

$\|$ Txt.4. sin color, solo la silueta negra | con un ... tagono tipo de "alto color rojo, | que decía en letras blancas "No más | Bullying". | Así como cuando vemos una señal de | alto, aún sin saber leer, quien ve esa $\mid$ señal se detiene. Así que cada vez $\mid N O^{4}$ que los alumnos pasaban $\mid M A S^{5}$ se detenían y leían. $\left|B U L L Y I N G^{6}\right|$ Cuando se iban comentaban unos | con otros, "viste acosar es hacer bullying" | o frases similares. | En resumen el mural fue todo un |éxito. Docentes y alumnos se detenían | a leer. |

En las últimas líneas del texto 3, la estudiante especifica mejor sobre la "actividad" del bullying mencionada en el texto 2. En este pasaje ella especifica que unos de los objetivos era que el alumnado de la escuela se detuviera delante del mural y lo viera, pero no explica cuál es el objetivo de la actividad o qué intenta motivar en el alumnado con eso.

Ella ofrece una descripción física detallada del mural, transcribe una reacción de un alumno y finaliza evaluando que todo fue un éxito. Aunque la estudiante haya descrito más detenidamente ese pasaje en su diario, las especificaciones no dan cuenta de movilizar los conocimientos de la carrera con lo vivido en la práctica por ser descripciones muy genéricas y que no caracterizan la especificidad docente, la trascripción de un habla en el diario es algo importante para ejemplificar con más pormenorización las situaciones que ocurren en la práctica.

La docente de la práctica podría apuntar que, partiendo de esa frase dicha por un alumno, la estudiante de la práctica pudiera hacer reflexiones más detenidas como por ejemplo, cuál fue el éxito que tuvo la actividad y justificarlo evidenciando apuntes que lo demuestren, pero se nota que la licenciada apenas le escribió "great", sugiriendo que sus apuntes estaban óptimos.

4 Parte del dibujo hecho en el texto para representar el cartel.

5 Parte del dibujo hecho en el texto para representar el cartel.

6 Parte del dibujo hecho en el texto para representar el cartel. 
Figura 5. Reproducción fac-similar de parte de diario de campo producido por una estudiante de la Práctica

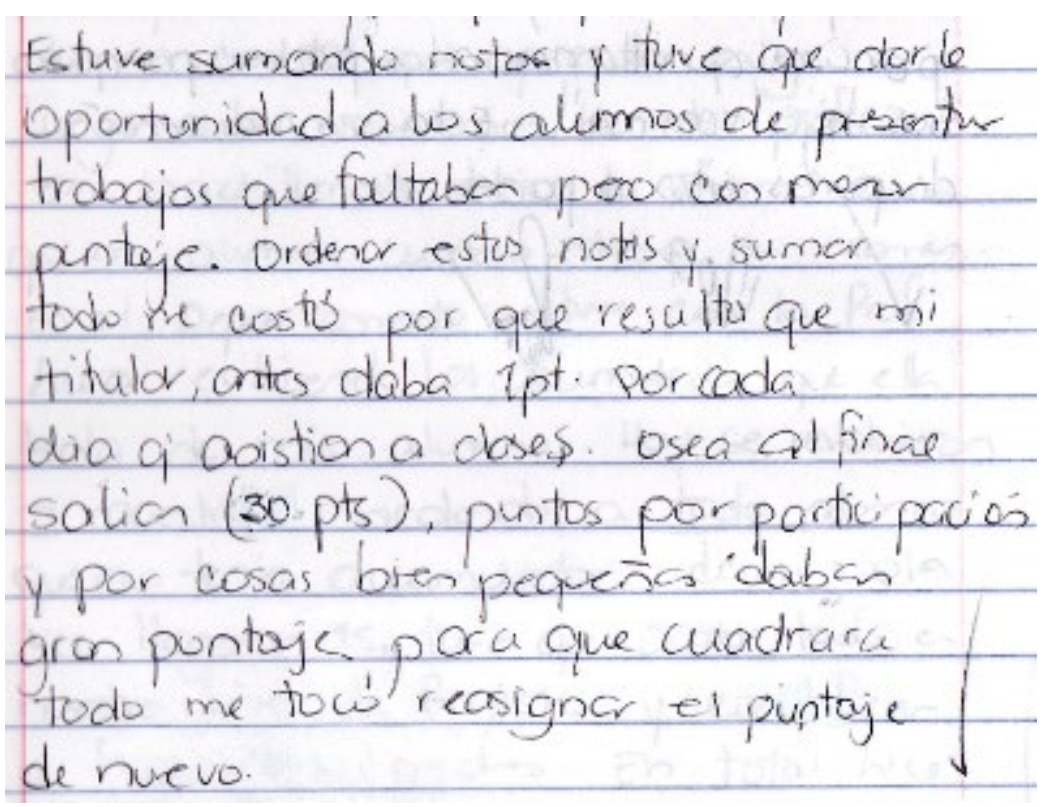

Transcripción del texto 5, producido por una estudiante de la Práctica II (en la Universidad Pedagógica Nacional Francisco Morazán, Tegucigalpa, Honduras)

||Txt.5. || Estuve sumando notas y tuve que darle | oportunidad a los alumnos de presentar $\mid$ trabajos que faltaban pero con menos $\mid$ puntaje. ordenar estas notas y sumar $\mid$ todo me costó por que resulto que mi | titular, antes daba 1 pt. por cada | dia q coistian a clases. osea al final | salian (30 pts), puntos por participación | y por cosas bien pequeñas daban | gran puntaje, para que cuadrara $\mid$ todo me tocó reasignar el puntaje | de nuevo.

Aquí se nota que la estudiante escribe con detalles que no le darían a las actividades retrasadas los mismos puntos que a las entregadas en la fecha correspondiente, sigue especificando aún sobre el puntaje y al fin del texto emite su opinión sobre ellos, aunque no ejemplifique lo que considera como cosas "bien pequeñas". 
Lo que llama la atención es que, en esta parte del diario, hay un especificación de detalles sobre las notas, pero en relación con los temas dados en clase o a algo que sea más detallado sobre los asuntos que ocurrieron en sala de clase, no. No hay mención, por ejemplo, de cómo eran los trabajos que presentaron sus estudintes o sobre cómo manejaban el tema presentado, ya que ella les evaluó.

Figura 6. Reproducción fac-similar de parte de diario de campo producido por estudiante de la Práctica

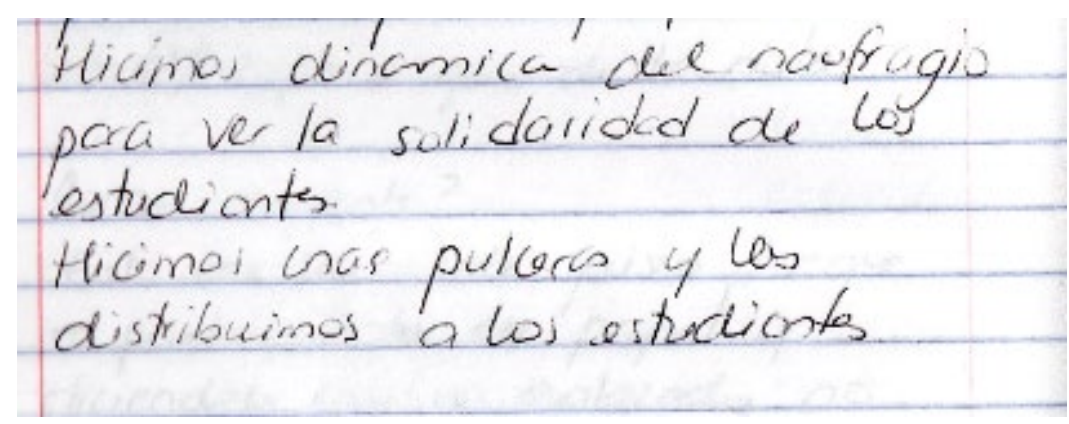

Transcripción del texto 6, producido por una estudiante de la Práctica II (en la Universidad Pedagógica Nacional Francisco Morazán, Tegucigalpa, Honduras)

||Txt.6. || Hicimos dinámica del naufragio | para ver la solidaridad de los $\quad$ estudiantes. | Hicimos unas pulceras y las $\mid$ distribuimos a los estudiantes |

En el texto 6 se nota que hay la mención del nombre de la actividad, naufragio, y el objetivo que tiene ella, ver la solidaridad de sus estudiantes.

Los textos 7 y 8 serán analizados conjuntamente porque están interconectados: 
Figura 7. Reproducción fac-similar de parte de diario de campo producido por una estudiante de la Práctica

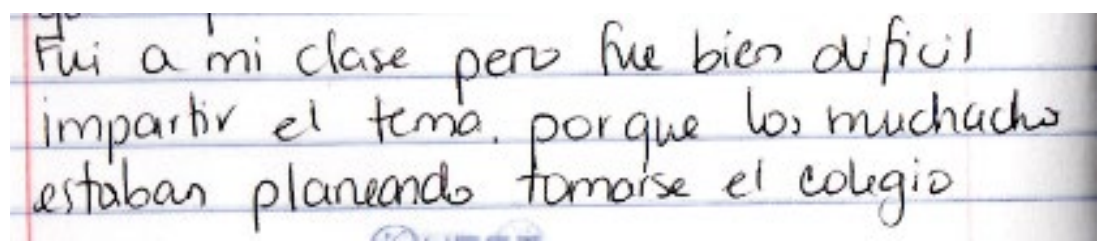

Transcripción del texto 7, producido por una estudiante de la Práctica II (en la Universidad Pedagógica Nacional Francisco Morazán, Tegucigalpa, Honduras)

||Txt.7. || Fui a mi clase pero fue bien dificil | impartir el tema. porque los muchacho | estaban planeando tomarse el colegio |

Figura 8. Reproducción fac-similar de parte de diario de campo producido por estudiante de la Práctica

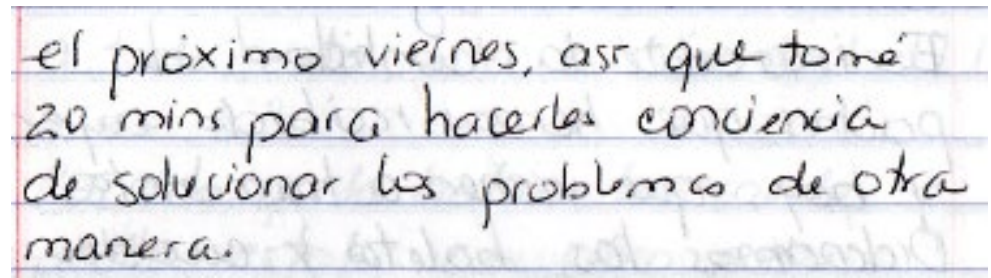

Transcripción del texto 8, producido por una estudiante de la Práctica II (en la Universidad Pedagógica Nacional Francisco Morazán, Tegucigalpa, Honduras)

||Txt.8. || el próximo viernes, así que tomé| 20 mins para hacerles conciencia $\mid$ de solucionar los problemas de otra $\mid$ manera $\mid$

En el texto 7 la estudiante relata que le resultó difícil impartir el tema, pero no informa qué tema fue impartido por ella y sigue explicando cuál fue la dificultad que le impidió cumplir con lo que había planeado.

En el texto 8 la alumna relata que, por motivo de la dificultad de proseguir con lo planeado, ella hizo una intervención de 20 minutos para "hacerles conciencia", pero no da a conocer qué ha dicho a sus estudiantes.

A partir del análisis de los escritos de estudiantes podemos mencionar, en primera instancia, que las dificultades más significativas que observamos fueron: 
- Aspectos contextuales

- Dificultad para expresar el uso adecuado de la contextualización.

- En sus escritos no utilizan citas ni referencias bibliográficas.

- Hablan de reflexión, pero no dan a conocer la reflexión.

- Aspectos referidos a la organización de la información

- Fallas en las diferentes partes que conforman un diario de campo, en lo cual se debe considerar que este es el escrito que presentan como requisito final.

- Además, no demuestran un proceso planificado en el cual considerar unos ejes temáticos orientadores de la observación durante la práctica profesional.

- $\quad$ No hay una descripción clara de las actividades realizadas durante la práctica profesional.

- $\quad$ Aspectos referidos a los contenidos

- $\quad$ No presentan ninguna relación de la teoría con la práctica.

- No presentan fundamento ni integración de planteos teórico-críticos.

- No asumen ninguna posición crítica.

- No presentan conclusiones.

- $\quad$ Aspectos referidos al uso de la lengua escrita

- No presentan fundamento ni integración de planteos teórico-críticos.

Además, se pudo observar la presencia de varios borradores o reescrituras por trabajo, con avances importantes en cada versión, en relación con las dificultades que iban presentando, pero faltan datos generales de la persona estudiante en el documento presentado. 


\section{Consideraciones finales}

Lo que necesita el estudiantado, ante las dificultades encontradas, es reorganizar los conocimientos del tema para lograr una escritura fundamentada. Sus docentes deben estimular la práctica de jerarquizar las ideas, organizar y estructurar su pensamiento, lo que no es una tarea sencilla. La comunicación oral no necesita de este ordenamiento, porque se conoce el lugar, la fecha y el destinatario del mensaje; al contrario, en la escritura, es necesario detallar ciertas informaciones para ubicar al público lector y lograr una mejor comunicación. El estudiantado necesita romper la barrera inicial de una escritura basada en su interpretación de la información, para una escritura que pretende comunicar su propósito a quien lo lee, estableciendo una relación entre la información y su comprensión sobre ella (Carlino, 2006).

Al observar los diarios, notamos que la mayoría, de hecho, sigue las preguntas disparadoras y contesta qué ha hecho el día de la práctica y cómo se sintió. Para una escritura inicial, esas preguntas pueden auxiliar al estudiantado al ingresar a su proceso de escritura, pero es necesario que el personal docente avance en las orientaciones, a fin de conducirlo a escrituras más específicas que dialoguen con el saber científico que proporciona la universidad para la construcción de saberes que su práctica les aporta.

En ese sentido, Carlino (2006) distingue dos tipos de escritura. El primer tipo de escritura como escritura privada, es una escritura llena de significados para quien la escribe; y el segundo tipo como escritura pública porque es pensada y organizada para un público. Sugiere que el estudiantado, primeramente, se ejercite mucho en la escritura privada para después dedicarse a la escritura pública, pero en la etapa de la privada hay que tener en cuenta que su escritura no puede quedarse meramente en esta, sino avanzar, es decir, después de su escrita privada, cada estudiante debe reescribir su escrito pensando en su público lector.

Nos parece que los diarios pueden ser considerados escrituras públicas porque el autor sabe quién lo leerá -no va a ser el personal directivo o docente del colegio, tampoco sus estudiantes, sino la persona académica de la universidad que orienta su práctica. Pero aún les falta más especificidad y movilización de los aportes teóricos en sus textos. Por otra parte, cabe al personal docente apuntar en dónde sus practicantes pueden avanzar en sus textos. 
En ese sentido, el personal docente les puede ayudar a desarrollar el pensamiento enseñando cómo encarar el texto de su asignatura, compartiendo la lectura con sus grupos y discutiendo fragmentos claves del texto. También es importante que el profesorado evidencie qué factores necesitan desarrollar y crear condiciones que favorezcan su desenvolvimiento. Si el profesorado tiene en cuenta que sus estudiantes enfrentan una nueva cultura y que para la mayoría es un gran desafío el proceso de integrarse a una comunidad distinta, puede ayudarles proponiendo estrategias para que logren introducirse a esa cultura paulatinamente. El personal docente tiene un importante papel como mediador, creando un puente entre estudiantes - la cultura que traen consigo - y la cultura de la academia. Sin embargo, no están en juego solamente las cuestiones del conocimiento y manejo de ello, sino también la inserción de un grupo hasta entonces externo hacia el interior de la cultura académica (Carlino, 2006).

Es fundamental proporcionar orientaciones pertinentes al estudiantado, esto es, el conjunto de estrategias que, de acuerdo con Cassany (1995 en Bono, Barrera,1998), "constituyen propiamente un buen proceso de composición escrita". De esta manera, se favorecerán sus escritos,

La enseñanza y el desarrollo de la escritura no solo corresponde a docentes de letras y lenguas; debe ser considerada desde cada uno de los espacios de todas las disciplinas; independientemente de la asignatura que se enseñe, se debe estimular el acto de escribir desde el contexto aúlico.

Los datos complementarios se refieren específicamente al trabajo de producción de textos académicos donde es frecuente que parte de lo que se escribe se refiera a otros textos académicos, razón por la cual es de suma importancia acceder a una adecuada comprensión lectora y desarrollar habilidades para resumir escritos.

Retomando las ideas expuestas al comenzar este trabajo y teniendo en cuenta las dificultades que efectivamente mostró el estudiantado en esta experiencia, podemos indicar un déficit en la utilización de estrategias cognoscitivas para la elaboración de trabajos escritos con el que se ingresa a la universidad, se hace evidente y se requiere de una temprana intervención pedagógica con el fin de orientar al alumnado en la realización de este tipo de tareas.

Recordemos, finalmente, que el desarrollo de la escritura no debe estar librado solamente a la puesta en acción de habilidades individuales. 
Teniendo en cuenta esto, debemos encontrar los espacios desde nuestro lugar de docentes, con el propósito de estimular el acto de escribir desde un contexto aúlico, favorecedor de acciones orientadas a la formación de un estudiantado universitario como productor de textos. Pasaremos así de la consideración de escribir como producto a la de escribir como proceso (Candlin, 1983).

\section{Referencias}

Bono, A. y Barrera, S. (Abril, 1997). Los estudiantes universitarios como productores de textos. Una experiencia de docencia compartida. Lectura y Vida, 19, 13-20.

Candlin, C. (1983). Learning to write. Freeman.

Carlino, (2003a). Leer textos científicos y académicos en la educación superior: Obstáculos y bienvenidas a una cultura nueva. $6^{\circ} \mathrm{Con}$ greso Internacional de Promoción de la Lectura y el Libro en las XIII Jornadas Internacionales de Educación. Fundación El Libro. Buenos Aires

Carlino, P. (Julio - agosto, 2004). El proceso de escritura académica: Cuatro dificultades de la enseñanza universitaria. Educere, 8(26), 321-327.

Carlino, P. (2006). La escritura en la investigación. Documentos de trabajo. Escuela de Educación de la Universidad de San Andrés. Argentina.

Cisneros, M., Olave, G. y Rojas, I. (2013). Alfabetización académica y lectura inferencial. Ecoe.

Cisneros, M., $\quad$ Olave, G. y Rojas, I. (2014). Cómo escribir la investigación académica. Desde proyecto hasta la defensa. Ediciones de la U.

Cassany, D. (1995). Describir el escribir. Paidós.

Lopez, G. y Arciniegas, E. (2007). La transformación de las prácticas de lectura y de escritura en la universidad desde una perspectiva metacognitiva. I Encuentro nacional sobre políticas institucionales para el desarrollo de la lectura y la escritura en la educación superior (pp. 1-11). U. Sergio Arboleda.

Moraes, D. M. A. y Fairchild, T. M. (2018). Discursos construidos: Narrativas escritas pelo profesor/estagiário. Recorte, 15(1), 1-18.

Pérez, M. y Rodríguez, A. (2013). ¿Para qué se lee y se escribe en la universidad colombiana? Caracterización de prácticas de 
lectura y escritura en 17 universidades. REDU. Revista de Docencia Universitaria, 11(1), 137-160. https://doi.org/10.4995/ redu.2013.5595

Spina, S. (1994). Introdução à edótica (2. ${ }^{\mathrm{a}}$ ed. rev. e atual). Ars Poetica; Edusp.

Zabalza, M. (2004). Diario de clase: Un instrumento de investigación y desarrollo. Profesional. NARCEA Ediciones. 